\title{
Probiotic protection of rotavirus gastroenteritis in an experimental rat model in early life
}

\author{
T. Pérez-Berezo, S. Ramos-Romero, C. Castellote, M. Castell, A. Franch and F. J. Pérez-Cano \\ Department of Physiology, Faculty of Pharmacy, University of Barcelona; Institut de Recerca en Nutrició i Seguretat \\ Alimentària (INSA-UB), Barcelona, Spain
}

Group A rotaviruses are the most common causative agents of acute gastroenteritis in children under two years. Previous reports suggest breast-feeding and the use of probiotics and prebiotics as protective agents for moderating the clinical course of rotavirus infection. The present study was designed to establish the utility of the suckling rat rotavirus infection model ${ }^{(1)}$ to evaluate the protective role of probiotics. From $3^{\text {rd }}$ day of life, Lewis neonatal suckling rats received daily $10 \mathrm{mg} / \mathrm{mL}$ of a particular bifidobacteria strain. On day $8^{\text {th }}$ of life, a heterologous simian rotavirus SA-11 was inoculated orally in the probiotic group and in a non-supplemented group. Rotavirus infection was evaluated daily by clinical indexes based on color, texture and amount of obtained feces. Fecal samples were used to quantify viral shedding and specific antibody production.A significant reduction in diarrhea incidence was observed in the probiotic group $(\sim 30 \%)$ when compared with the non-supplemented group $(\sim 70 \%)$ on day 4 after infection $(p<0.05)$. The length of the diarrhea period was also shortened by one day. The disease severity was controlled by the probiotic as is shown by lower severity curve with respect to the non-supplemented animals (AUC of $\sim 6.1 \mathrm{vs}$ AUC of $8.4, p<0.05$ ) although the viral shedding and specific antibody production pattern was not significantly affected. In conclusion, these results demonstrate the suitability of this model to evaluate the reduction of the incidence and severity of a rotavirus-induced acute gastroenteritis by a daily supplement such as a probiotic.

1. Pérez-Cano FJ, Castell M, Castellote C et al. (2007) Pediatr Res 62, 658-663. 